\title{
Journal of

\section{Shear stiff, surface modified, mica-like nanoplatelets: a novel filler for polymer nanocomposites}

\author{
Michael R. Schütz, ${ }^{a}$ Hussein Kalo, ${ }^{a}$ Thomas Lunkenbein, ${ }^{a}$ André H. Gröschel, ${ }^{c}$ Axel H. E. Müller, ${ }^{c}$ \\ Charles A. Wilkie ${ }^{b}$ and Josef Breu ${ }^{* a}$
}

Received 6th April 2011, Accepted 1st June 2011

DOI: $10.1039 / \mathrm{c} 1 \mathrm{jm} 11443 \mathrm{c}$

Synthesis of polymer nanocomposites with novel shear stiff, mica-like nanoplatelets from a synthetic layered silicate is presented. This novel synthetic clay filler shows high aspect ratios while organophilization may be selectively restricted to external surfaces minimizing the organic content of the filler. The obtained nanocomposite shows superior mechanical, thermal and fire properties as compared to commonly used natural clays. Furthermore, the influence of the blending method on the nanocomposite properties was investigated.

\section{Introduction}

Almost twenty years ago the pioneering findings of Toyota researchers initiated the study of polymer nanocomposites. ${ }^{1,2}$ Since then, all kinds of nanoscopic fillers and polymers have been blended to yield hybrid materials with enhanced properties. Mechanical, electrical, thermal, barrier, and flammability properties could be improved with different kinds of fillers. In particular, research in the field of clay nanocomposites exploded because of the cheap and modifiable nature of the layered silicates. $^{3}$

A significant drawback of these natural clay fillers is that cation exchange cannot be tuned to be selective. Organophilization by cation exchange always affects both internal and external surface cations concomitantly. ${ }^{4}$ Intercalated organic cations, however, represent inner shear planes possibly inducing failure at the interlayer when introducing shear stress. ${ }^{5}$

A shear stiff, mica-like clay where all interlamellar spaces are crosslinked should therefore be advantageous, ${ }^{6}$ in particular for mechanical reinforcement and flame retardancy applications.

Various polymer matrices and diverse clays have been used to synthesize flame retardant nanocomposites. ${ }^{7}$ The mechanism by which smectite type clays can influence the burning behavior of nanocomposites has been elucidated. During the degradation of the polymer, a clay barrier is formed on the surface of a nanocomposite which inhibits mass transfer and insulates the underlying polymer from radiated energy. ${ }^{8,9}$ The main prerequisites to achieve these improvements are homogeneously distributed

${ }^{a}$ Lehrstuhl für Anorganische Chemie I, Universität Bayreuth, 95440 Bayreuth, Germany. E-mail: josef.breu@uni-bayreuth.de; Fax: +49 921552788; Tel: +49 921-552531

${ }^{b}$ Department of Chemistry and Fire Retardant Research Facility, Marquette University, P.O. Box 1881, Milwaukee, WI, 53201, USA

${ }^{c}$ Lehrstuhl für Makromolekulare Chemie II, Universität Bayreuth, 95440 Bayreuth, Germany fillers, i.e. high degree of dispersion in the polymer matrix. Modification of the surface tension of the filler fosters good dispersion and helps maximize the specific interface between matrix and filler. Different blending or in situ methods were used to mix and exfoliate the clay in one step. ${ }^{10}$

Additionally, particle size and particle shape have a big influence on the barrier properties. For anisotropic fillers, such as clay platelets, the aspect ratio, i.e. the diameter divided by the thickness of the platelet, is crucial to achieve enhanced properties. ${ }^{11}$ Tamura et.al. ${ }^{12}$ developed a method where natural mica is exfoliated to yield clay fillers with high aspect ratios which are inherited from the huge diameter of the pristine mica platelets. However, the exchange of the interlayer cations is quite difficult and slow, additionally a large excess of the organic modifier was required because mica shows only very limited intracrystalline reactivity.

In this study we apply novel, shear stiff, mica-like nanoplatelets as fillers in polystyrene (PS) nanocomposites for flame retardancy. This mica-like material shows huge aspect ratios and is produced by a fast and facile process from a synthetic Nafluoro-hectorite. ${ }^{6}$ Moreover, these synthetic clays can exclusively be modified on the outer basal particle surface. This is a major advantage of these clays over commonly used natural clays like montmorillonite (MMT) since the easy inflammable organic content of the filler is significantly reduced.

\section{Experimental section}

\section{Materials}

Polystyrene (PS, $M_{\mathrm{w}} \approx 192000 \mathrm{~g} \mathrm{~mol}^{-1}$, melt index $6.00-9.00 \mathrm{~g}$ per $10 \mathrm{~min}\left(200{ }^{\circ} \mathrm{C}\right.$ per $5.0 \mathrm{~kg}$, ASTM D1238)), acetic acid, toluene, tetrahydrofuran (THF), methanol, 2,2'-azobis(2-methylpropionitrile) (AIBN), 1-dodecanethiol, and monomers (dodecyl methacrylate and 2-(dimethylamino)ethyl 
methacrylate, DMAEMA) were purchased from Aldrich Chemical Co. The Na-fluoro-hectorite used was synthesized according to a reported procedure. ${ }^{13}$ The hectorite showed the following idealized formula unit: $\mathrm{Na}_{0.6}\left(\mathrm{Mg}_{2.4} \mathrm{Li}_{0.6}\left[\mathrm{Si}_{4} \mathrm{O}_{10} \mathrm{~F}_{2}\right]\right)$. The inorganic salts $\left(\mathrm{MgCl}_{2}, \mathrm{KCl}\right)$ used for cation exchange were purchased from Grüssing GmbH Analytika, Germany. All chemicals were used without further purification, unless otherwise noted.

\section{Instrumentation and characterization}

Exfoliation of the hectorite was carried out in a stirred media mill (Netzsch Labstar LS 1 LMZ) with stainless steel equipment. The grinding media were yttrium stabilized zirconia beads with a diameter of $2 \mathrm{~mm}$. The volume fraction of the grinding media in the grinding chamber was $80 \mathrm{vol} \%$. The $10 \mathrm{wt} \%$ aqueous hectorite dispersion was wet ground for two hours in a closed loop (flow rate: $50 \mathrm{~kg} \mathrm{~h}^{-1}$ ).

The particle size was determined by static light scattering using a Retsch Horiba LA-950 SLS instrument. The solid content of the dispersions was adjusted to yield a transmission of $80 \%$ of the laser intensity. The calculation of the particle size distribution was performed according to the theory of Fraunhofer diffraction.

Powder X-ray diffraction (PXRD) measurements were obtained using a Rigaku Miniflex II Desktop X-ray diffractometer (Bragg-Brentano geometry) with $\mathrm{Cu}\left(\mathrm{K}_{\alpha}\right)$ radiation $\lambda=$ $1.54078 \AA$, from a sealed X-ray tube. The pestled powder samples were prepared and mounted on a glass holder. The data were collected at $2 \theta$ values varying from $5^{\circ}$ to $70^{\circ}$ at a scan speed of $5^{\circ}$ per minute with a sampling width of 0.02 .

The melt compounded hectorite PS composite (total amount $50 \mathrm{~g}$ ) was mixed using a Brabender mixer at $180{ }^{\circ} \mathrm{C}$ for $10 \mathrm{~min}$ at a screw speed of $60 \mathrm{rpm}$.

The specimens for the mechanical analysis were prepared by injection molding. The samples were melted $\left(190^{\circ} \mathrm{C}\right)$ and pressed into a dog-bone shaped mold $\left(30^{\circ} \mathrm{C}, 0.8 \mathrm{MPa}\right)$. The mechanical properties were determined according to ISO 527 at room temperature in an Instron 5565 universal tester. The mean values of at least seven measurements are reported. Moduli were determined at a strain rate of $0.2 \mathrm{~mm} \mathrm{~min}^{-1}$; other values were obtained at a rate of $1 \mathrm{~mm} \mathrm{~min}^{-1}$.

Scanning electron microscope (SEM) images were recorded with a Zeiss 1530 FESEM with an acceleration voltage of $5 \mathrm{kV}$. The samples were freeze-dried, mounted on conductive sample holders and sputtered with carbon.

Transmission electron microscope (TEM) measurements were carried out on a Zeiss EM 922 EFTEM with an acceleration voltage of $200 \mathrm{kV}$. Specimens were microtomed to obtain 30-70 $\mathrm{nm}$ thick pieces, which were placed on a lacey carbon copper grid.

Thermogravimetric analyses (TGA) were conducted with a Netzsch TG209 F1 thermoanalyzer instrument. Specimens with a mass of $15 \pm 1 \mathrm{mg}$ were heated from 30 to $700{ }^{\circ} \mathrm{C}$ at a heating rate of $10{ }^{\circ} \mathrm{C} \mathrm{min}-1$ in a nitrogen atmosphere at a flow rate of $20 \mathrm{ml} \mathrm{min}{ }^{-1}$. All samples were run in duplicate and the average values are reported; the temperature is reproducible to $\pm 2{ }^{\circ} \mathrm{C}$ and the mass to $\pm 0.4 \%$.
The cone calorimeter experiments were carried out using an Atlas Cone 2 instrument according to ASTM E 1354, on $3 \mathrm{~mm}$ thick $100 \times 100 \mathrm{~mm}^{2}$ plaques. All samples were tested in triplicate. The cone data obtained are reproducible to within $\pm 10 \%$ when measured at a heat flux of $35 \mathrm{~kW} \mathrm{~m}^{-2}$.

\section{Sample preparation}

The sodium hectorite was dispersed in deionized (DI) water (about $3 \mathrm{wt}^{\mathrm{o}} \%$ ). All following steps were carried out without drying. Standard procedures were used to exchange the $\mathrm{Na}^{+}$ cations with $\mathrm{Mg}^{2+} \cdot{ }^{14}$ After ion exchange, the hectorite was centrifuged and washed several times with DI water until the wash water showed no reaction with $\mathrm{AgNO}_{3}$.

The Mg-hectorite was redispersed in DI water to yield a solid content of $10 \mathrm{wt} \%$. This dispersed $\mathrm{Mg}$-hectorite was exfoliated in a stirred media mill. Subsequently, the $\mathrm{Mg}^{2+}$ ions were exchanged with $\mathrm{K}^{+}$ions. The $\mathrm{K}$-hectorite was centrifuged and washed to yield a $\mathrm{K}$-hectorite dispersion with a solid content of $3 \mathrm{wt} \%$. This dispersion was used as a starting dispersion for all PS composites.

The oligomeric modifier was synthesized via free radical polymerization with AIBN (7.5 mmol) as initiator and 1dodecanethiol as chain transfer agent. Prior to polymerization, the inhibitor of the monomers was removed by passing the monomers over basic alumina. The statistical copolymerization was carried out after three freeze-pump-thaw cycles with a ratio of AIBN : monomer : 1-dodecanethiol = $1: 50: 2$ and DMAEMA : dodecylmethacrylate $=1: 4$. The reactants were dissolved in $100 \mathrm{ml}$ toluene and the mixture was reacted for 90 min under reflux conditions. Afterwards, the oligomeric modifier was precipitated in 31 methanol and recovered by centrifugation of the methanolic solution. The resulting statistical copolymer shows a mean composition of DMAEMA : LaurylMA $=1: 5.44$ with $M_{\mathrm{n}}=3860 \mathrm{~g} \mathrm{~mol}^{-1}$, PDI $=1.6$, determined by ${ }^{1} \mathrm{H}$ NMR and GPC.

The K-hectorite dispersion was flocculated with a water/THF $(1: 1 \mathrm{v} / \mathrm{v})$ dispersion of the oligomeric modifier. Here, the modifier was dissolved in THF and an equimolar amount of acetic acid was added to quaternize the amine functions. Subsequently, the solution was mixed with DI water to yield the oligomeric modifier dispersion. This dispersion was combined with the K-hectorite dispersion until all of the hectorite was flocculated. Subsequently, the surface modified K-hectorite was filtered and washed several times with DI water and water/THF mixtures. Next, the hectorite was redispersed in toluene $(200 \mathrm{ml})$ or dried at room temperature depending on the blending method applied for composite formation.

For the solution blended nanocomposites $100 \mathrm{~g}$ of the PS was dissolved in $400 \mathrm{ml}$ toluene. Then, the dispersed hectorite in toluene was added to the PS solution. The amount of the hectorite dispersion was calculated to yield an inorganic content of $3 \mathrm{wt} \%$ in the dried nanocomposite. The PS modified hectorite dispersion in toluene was stirred rapidly for 12 hours then the toluene was allowed to evaporate at room temperature followed by evaporation in a vacuum oven at $80{ }^{\circ} \mathrm{C}$ for 36 hours.

The melt blended sample was prepared by mixing the dried surface modified K-hectorite and the PS in a melt mixer. Afterwards, the nanocomposites were melt pressed to yield plaques for the cone calorimeter experiments. 


\section{Results and discussion}

\section{Preparation of the novel filler}

Shear-stiff, mica-like nanoplatelets were obtained from a synthetic Na-hectorite by a recently published process by Möller et $a l .{ }^{6}$ The key to this process is a sensible tuning of the layer charge that allows switching between hydrated and dehydrated forms by simple cation exchange (Scheme 1). While the highly hydrated $\mathrm{Mg}$-form represents shear-labile material that can be exfoliated efficiently by applying shear forces, for instance in a stirred media mill, the K-form collapses to a mica-like shearstiff material. The degree of exfoliation and hence the thickness of the nanoplatelets may be controlled by the total shear force applied (milling time). Moreover, since the collapsed interlayers of the $\mathrm{K}$-form are no longer readily accessible for cation exchange, organic cations applied to modify the surface tension will only be exchanged at the external basal surfaces of the platelets. Please note that due to the collapse intercalation into the interlamellar space no longer is possible. We used an oligomeric modifier shown in Fig. 1. The exchange of inorganic $\mathrm{K}^{+}$by this oligomer at the external clay surface leads to an organophilization of the clay platelets. Consequently, the hectorite nanoparticles are destabilized in water and flocculate. This voluminous precipitate can easily be filtered and redispersed in organic solvents, where the interactions between the modifier and the organic solvent stabilize the suspension of particles again. Alternatively, the filtered nanoparticles can be dried for subsequent melt blending (Scheme 1).

Please note that with commonly used natural MMTs even the $\mathrm{K}$-forms are not collapsed but hydrated due to the much lower layer charge per $\mathrm{Si}_{4} \mathrm{O}_{10}$ unit ( $x=0.36$ and $x=0.60$, respectively). Consequently, the total cation exchange capacity (typically $90 \mathrm{mmol}$ per $100 \mathrm{~g}$ ) of internal and external surfaces of MMT is satisfied by the organic modifier. While the total exchange capacity of the pristine Na-hectorite is higher (136 mmol per

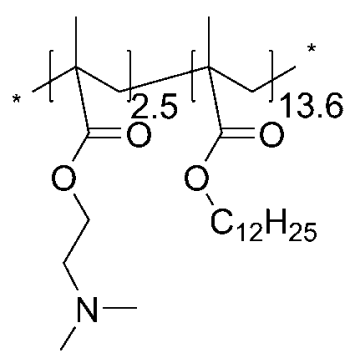

Fig. 1 Structure of the synthesized oligomeric modifier.

$100 \mathrm{~g}$ ), the exchange capacity of the external surfaces of a typical tactoid of $30 \mathrm{~nm}$ thickness is only $5 \mathrm{mmol}$ per $100 \mathrm{~g}$. Accordingly, the novel mica-like filler contains only a fraction of organic modifier of a natural MMT. Since it is well known that these organic modifiers are easily inflamed when applying these nanofillers as flame retardant additives in polymers, we expect this novel material to be superior to MMT.

\section{Characterization of the mica-like filler}

The synthesized mica-like K-hectorite platelets were freeze-dried to investigate the aspect ratio after the milling and cation exchange reaction. Fig. 2 shows a SEM image of the high aspect ratio clay platelets. These platelets show diameters up to $10 \mu \mathrm{m}$ with a thickness generally smaller than $50 \mathrm{~nm}$ (exact thickness is also determined by TEM in the PS nanocomposite, see below). However, the typical aspect ratio of the novel shear stiff clay filler is 200 or even higher.

As discussed above, the organophilization of the K-hectorite platelets is done by flocculation with the synthesized modifier. Proof of the exclusive modification of external surfaces by PXRD measurements was carried out investigating the K-hectorite before and after the organophilization (Fig. 3). The powder
I.

Exfoliation,
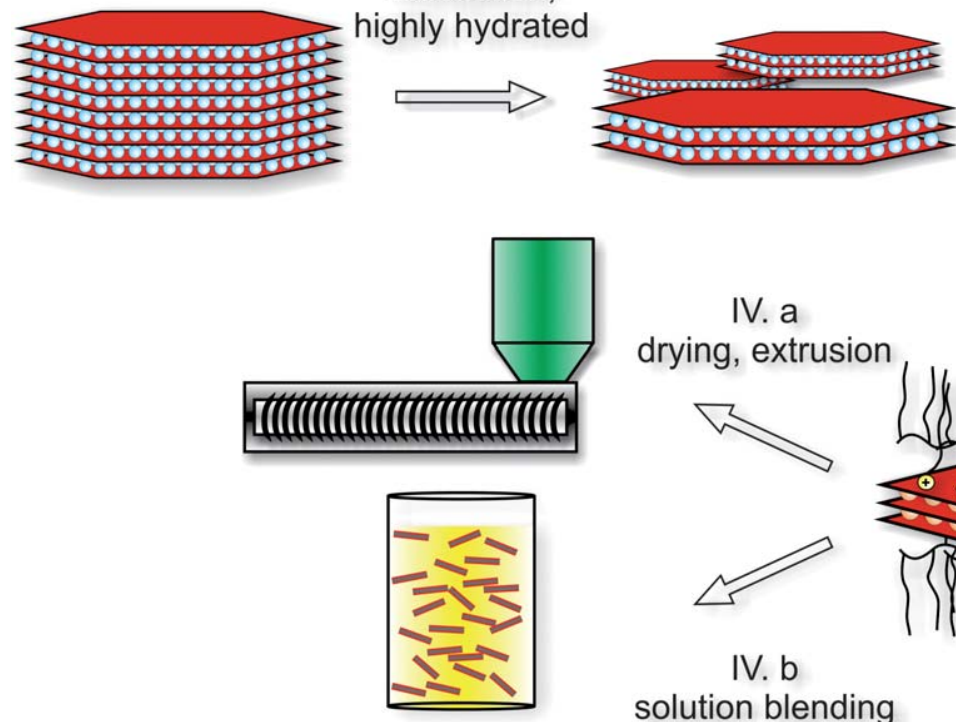

\section{drying, extrusion}

II. cation exchange, dehydration

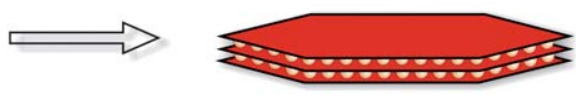

III. flocculation

Scheme 1 Preparation of the PS nanocomposite containing surface modified K-hectorite. 


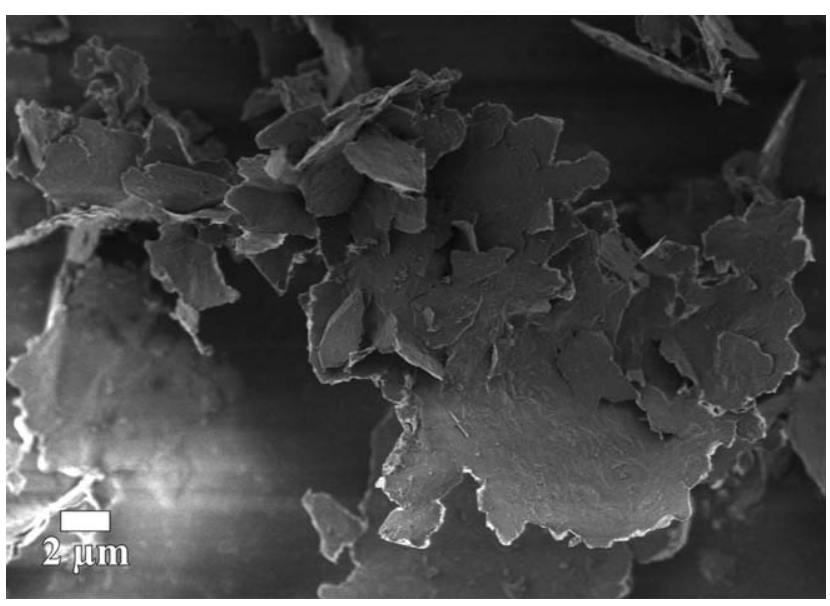

Fig. 2 SEM image of the exfoliated, freeze dried K-hectorite platelets.

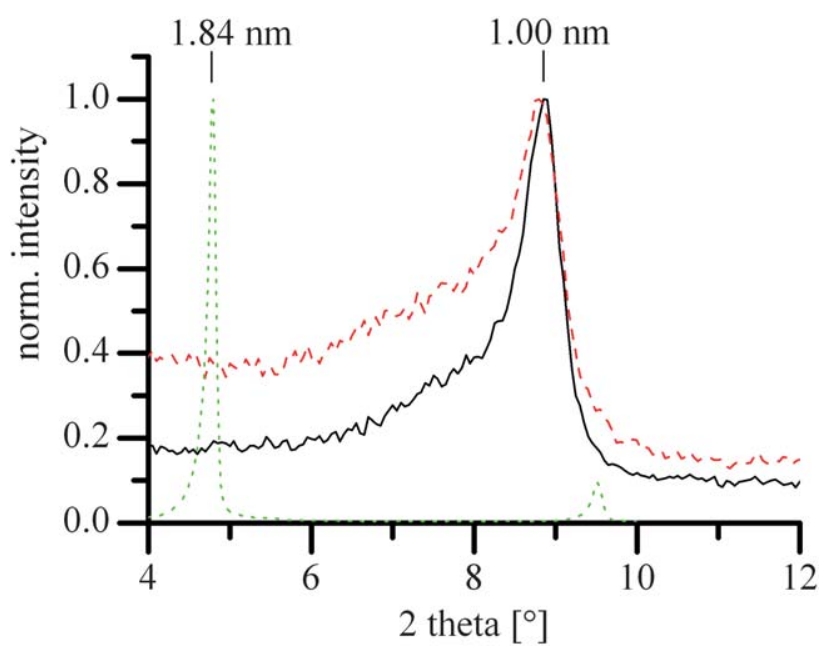

Fig. 3 PXRD pattern of the highly hydrated starting material $(\mathrm{Mg}-$ hectorite, $\cdots$ ), the dispersed K-hectorite before (-) and after (---) the surface modification.

X-ray diffraction (PXRD) pattern of organophilized K-hectorite shows a basal spacing of $1.00 \mathrm{~nm}$ which is typical for nonhydrated, mica-like 2:1 layered silicates. The interlayers are collapsed because of the low hydration energy of the $\mathrm{K}^{+}$cation. For comparison the diffraction pattern of the highly hydrated, shear-labile $\mathrm{Mg}$-form of the hectorite before milling is also shown. The basal spacing of the Mg-form is $1.84 \mathrm{~nm}$ indicating a 3-layer hydrate. After exfoliation by milling in a stirred media mill and subsequent cation exchange, the 001 reflection is significantly broadened with full widths at half maximum (FWHM) of $0.12^{\circ} v s .0 .79^{\circ} 2 \theta$, respectively, indicating efficient splitting of platelets into thinner tactoids.

Comparing the two basal reflections of the K-hectorites before and after surface modification with the oligocation, no shifts or significant further broadening can be detected. Hence, the oligomeric modifier does not intercalate into the interlayers of the mica-like platelets but is solely bound to the external surface of the platelets. Consequently, inorganic, shear stiff, high aspect ratio nanoplatelets with an oligomeric modifier on the surface were synthesized.
One prerequisite for a superior performance of these mica-like fillers is the maximization of the specific interface between matrix and filler. Thus prevention of agglomeration during the synthesis of nanocomposites is essential. Therefore, the change in the particle sizes during the dispersing and exchange steps is monitored by static light scattering. This measurement cannot show the degree of exfoliation but it is able to detect agglomeration since particle sizes determined for suspensions of anisometric clay platelets correlate well with platelet diameters. Fig. 4 shows the particle size distributions at different steps of the preparation. Prior to milling, Mg-hectorite has a broad particle size distribution with particle sizes ranging between $5 \mu \mathrm{m}$ and $100 \mu \mathrm{m}$. During the exfoliation in the mill the particle size decreases to yield particle sizes between $1 \mu \mathrm{m}$ and $10 \mu \mathrm{m}$. Besides disaggregation, most likely the diameter of the clay platelets also slightly decreases. Despite the pronounced anisotropy in bonding along and perpendicular to the clay lamellae, advanced exfoliation goes along with a partial breakage of the platelets. Nevertheless, clay platelets with very large aspect ratios can be produced. After the cation exchange of $\mathrm{Mg}^{2+}$ with $\mathrm{K}^{+}$the particle size distribution hardly changes. Thus, the change in the interlayer cation only induces collapse of interlayers but does not produce agglomerates. More surprisingly, even after the flocculation with the oligomeric modifier and the redispersion in organic solvents (here toluene) the particle size remains unchanged. With this result in mind we state that the excellent electrostatic stabilization of the clay platelets in water can be converted to an efficient steric stabilization in the organic solvent with the chosen modifier.

\section{TEM characterization of the PS nanocomposites}

The homogeneity of the dispersion of the novel surface modified clay filler in the PS matrix is determined by TEM images. Fig. 5 shows the TEM micrograph of the solution blended nanocomposite. No agglomerates are detected and the clay tactoids are distributed homogeneously in the PS matrix. Typical aspect ratios may be calculated from the diameter and the thickness of the platelets, which can be evaluated from the TEM images; the diameter of the platelets shown in Fig. 5 ranges between $1 \mu \mathrm{m}$

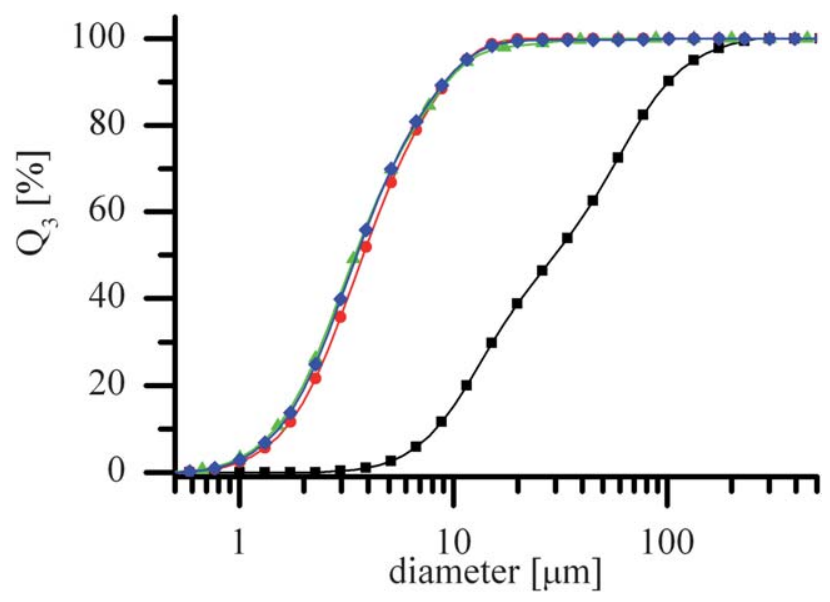

Fig. 4 Particle size distributions of the different hectorites: Mg-hectorite prior $(\boldsymbol{\square})$ and after milling $(\bullet)$, K-hectorite $(\boldsymbol{\Delta})$, surface modified Khectorite $(\bullet)$. 

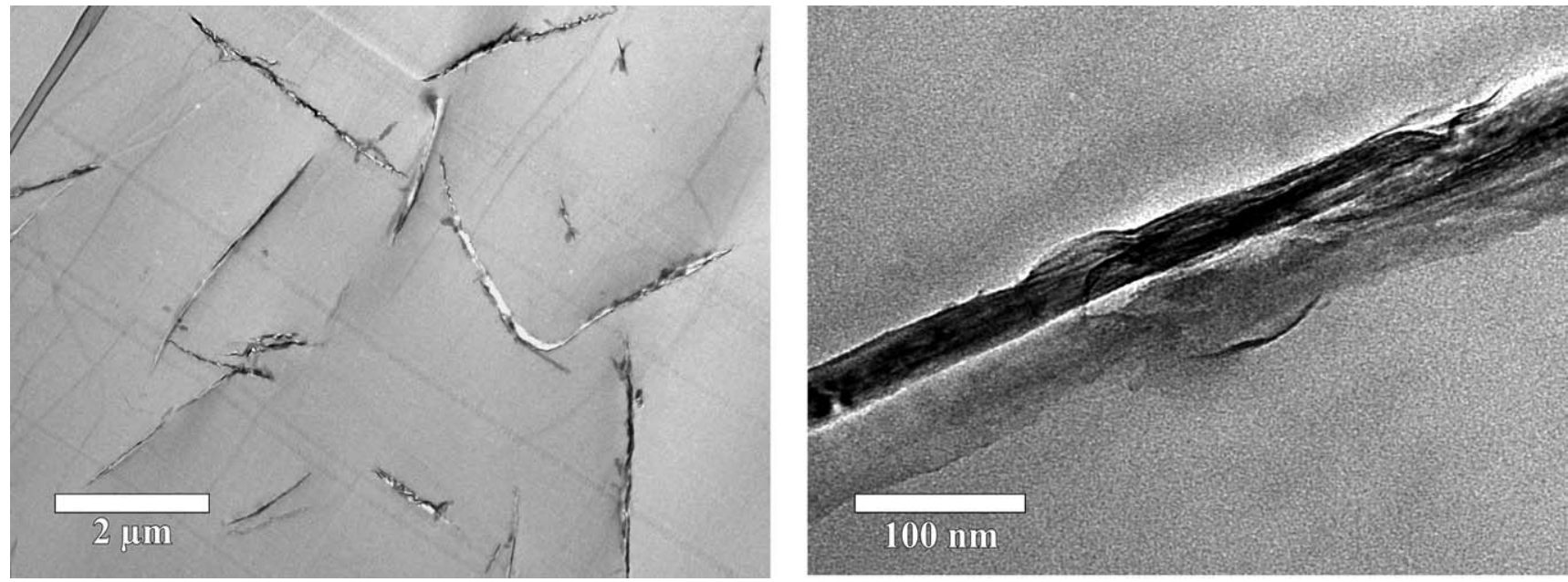

Fig. 5 TEM images of the solution blended PS surface modified K-hectorite nanocomposite at low (left) and high (right) magnification.

and $10 \mu \mathrm{m}$ and the thickness is approximately $30 \mathrm{~nm}$. Therefore, the determined aspect ratios range up to 350 . This result is in close agreement with the values derived from static light scattering experiments and SEM images. Fig. 6 shows a TEM micrograph of the melt blended PS surface modified K-hectorite. Comparing the two blending methods one can state that the drying of the surface modified K-hectorite induces some agglomerates and these agglomerates are not completely destroyed by melt mixing of the nanocomposites. Consequently, melt blending gives a much lower specific interface between matrix and filler, which is directly connected to mechanical and thermal properties of the nanocomposite.

In summary, this novel route leads to high aspect ratio platelets, which can be homogeneously dispersed by solution blending in PS. For melt blending the shear energy introduced during blending of the nanocomposite is not sufficient to completely destroy the agglomerates. However, it is expected that milder drying methods such as freeze drying and blending with higher shear energies should be capable to further reduce the

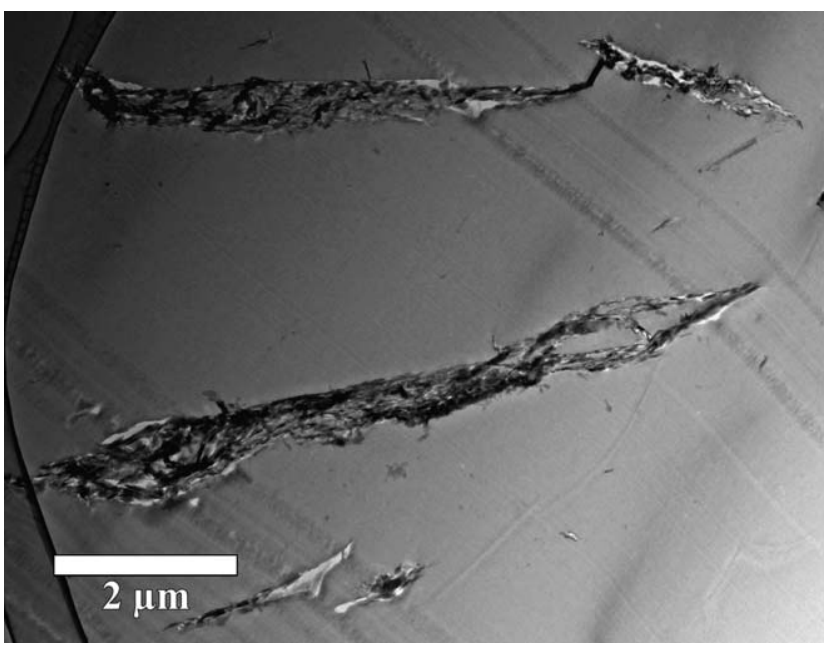

Fig. 6 TEM image of the melt blended PS surface modified K-hectorite nanocomposite at low magnification. agglomerates in melt blended nanocomposites. Please note that despite the huge aspect ratios, the thickness of the platelets is in the range of typical fundamental particles of MMT. This in combination with the good dispersion will assure specific interface areas between filler and polymer which are well comparable with typical MMT composites and which justify to call our composites nanocomposites.

\section{Mechanical properties of the PS nanocomposites}

The mechanical properties of the PS surface modified K-hectorite nanocomposites were evaluated by tensile testing (Table 1). Due to the stiff filler, Young's modulus of the solution blended nanocomposite increases (16\%) compared to the pure PS sample. The solution blended sample shows slightly better mechanical properties, which can be attributed to the absence of agglomerates leading to a maximized specific interface between matrix and filler. The strength at break and the strain at break do not change significantly for the nanocomposites compared to the pure PS sample. In summary, especially the solution blended PS surface blended nanocomposite shows a higher stiffness, whereas the values at break are almost the same compared to the PS control.

\section{Thermal properties of the PS nanocomposites}

The thermal properties of the oligomeric modifier, the novel surface modified clay filler, and the nanocomposites were determined by TGA measurements in $\mathrm{N}_{2}$ and the data are collected in Table 2. Fig. 7 shows that the pure oligomeric modifier

Table 1 Mechanical properties of the synthesized PS surface modified K-hectorite nanocomposites

\begin{tabular}{llll}
\hline & $\begin{array}{l}\text { Young's modulus/ } \\
\text { MPa }\end{array}$ & $\begin{array}{l}\text { Strength at } \\
\text { break/MPa }\end{array}$ & $\begin{array}{l}\text { Strain at } \\
\text { break (\%) }\end{array}$ \\
\hline $\begin{array}{l}\text { Pure PS } \\
\begin{array}{l}\text { PS nanocomposite } \\
\text { (solution) }\end{array}\end{array}$ & $1697 \pm 31$ & $47 \pm 4$ & $4.4 \pm 0.8$ \\
$\begin{array}{l}\text { PS nanocomposite } \\
\text { (melt) }\end{array}$ & $1908 \pm 21$ & $49 \pm 2$ & $3.7 \pm 0.3$ \\
\hline
\end{tabular}


Table 2 TGA data of the synthesized products

\begin{tabular}{llll}
\hline & $T_{0.1}{ }^{a} /{ }^{\circ} \mathrm{C}$ & $T_{0.5} /{ }^{\circ} \mathrm{C}$ & Char $(\%)$ \\
\hline Oligomeric modifier & 271 & 368 & 0 \\
Modified clay filler & 331 & - & 83.5 \\
PS control & 369 & 393 & 0 \\
PS nanocomposite (solution) & 380 & 410 & 2.7 \\
PS nanocomposite (melt) & 376 & 409 & 3.3
\end{tabular}

${ }^{a} T_{0.1}$, temperature at $10 \%$ mass loss; $T_{0.5}$, temperature at $50 \%$ mass loss.

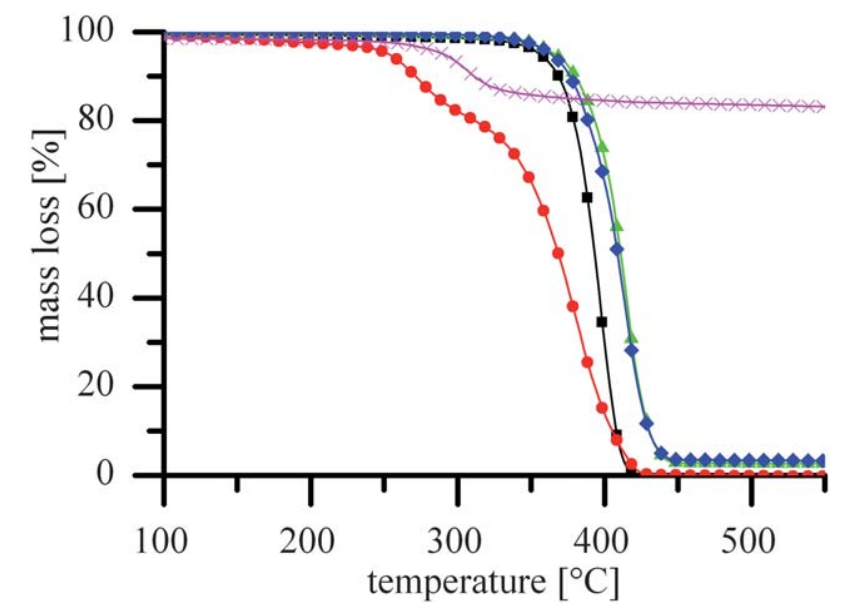

Fig. 7 TGA of oligomeric modifier ( $)$, surface modified K-hectorite $(\times)$, pure PS $(\boldsymbol{\square})$, solution blended PS nanocomposite $(\boldsymbol{\Delta})$, and melt blended PS nanocomposite $(\bullet)$.

decomposes in two steps. The onset temperature of degradation, herein defined as the temperature at which $10 \%$ degradation occurs $\left(T_{0.1}\right)$, of the pure oligomeric modifier is $271{ }^{\circ} \mathrm{C}$. This value is $100{ }^{\circ} \mathrm{C}$ lower than the onset of the pristine PS sample $\left(369^{\circ} \mathrm{C}\right)$ but much higher than the processing temperature during melt blending $\left(180{ }^{\circ} \mathrm{C}\right)$. Therefore, the modifier should not be degraded during the synthesis of the nanocomposite. Both PS surface modified K-hectorite nanocomposites show slightly enhanced thermal stabilities compared to the pristine PS sample. The onset temperature of the solution blended nanocomposite is increased by $11{ }^{\circ} \mathrm{C}$, whereas the onset of the melt blended nanocomposite is shifted by $7{ }^{\circ} \mathrm{C}$. Compared to pure PS, the temperature at $50 \%$ degradation $\left(T_{0.5}\right)$ is increased by $17^{\circ} \mathrm{C}$ and $16^{\circ} \mathrm{C}$, respectively. Therefore, the clay platelets are able to retard the decomposition of the PS matrix and the nanocomposites show a higher thermal stability. The inorganic content of the nanocomposites can be determined by the amount of residue at $500{ }^{\circ} \mathrm{C}$. Both nanocomposites show a residue of about $3 \mathrm{wt} \%$,

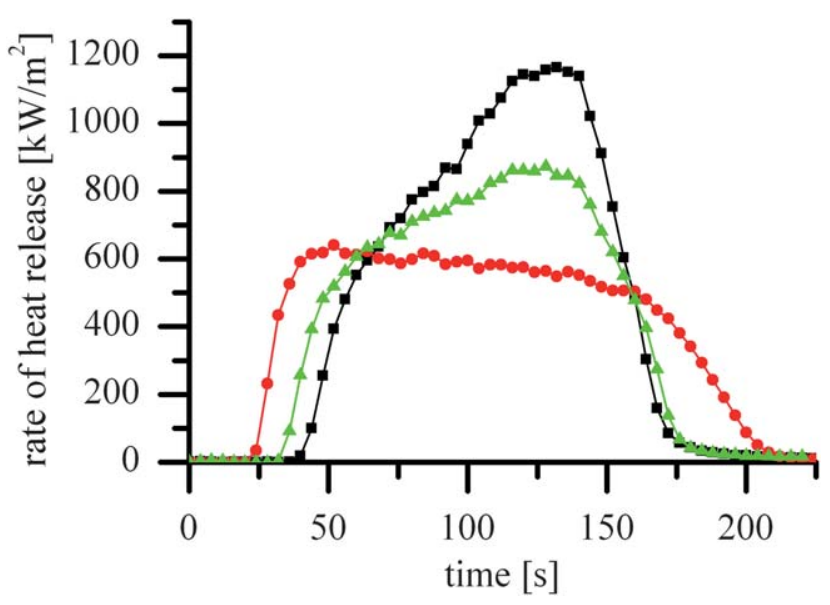

Fig. 8 Comparison of heat release rates (HRRs) for pure PS ( $\mathbf{a})$, solution blended K-hectorite $(\mathbf{O})$ nanocomposite and melt blended $\mathrm{K}$ hectorite $(\boldsymbol{\Delta})$ nanocomposite.

which matches the amount of inorganic clay mixed with the polymer during preparation of the composites. Hence, no additional carbonaceous char is formed during the decomposition of the PS matrix.

Moreover, due to the limitation of organophilization to the external surfaces, the inorganic content $(83.5 \mathrm{wt} \%)$ of the surface modified hectorite filler itself is much higher as compared to commercial MMT based fillers. $\mathrm{MMT}^{15}$ or ammoniumexchanged mica ${ }^{12}$ shows inorganic contents as low as $40 \mathrm{wt} \%$ when fully exchanged with $e . g$. hydrogenated tallow ammonium cations. This implies that the additional amount of easily inflammable organics added by the clay filler to the nanocomposite is reduced by up to $50 \mathrm{wt} \%$. Additionally, this novel type of clay filler offers the possibility to use more expensive or sophisticated organic modifiers for clay nanocomposites in different applications because significantly less modifier is needed, as has been pointed out.

\section{Flammability of the nanocomposites}

The fire properties of the nanocomposites were measured by cone calorimetry, the results are reported in Table 3 and typical curves are shown in Fig. 8. The nanocomposites provide distinctive results for layered silicate flame retardant materials. ${ }^{10}$ While the time of ignition $\left(t_{\mathrm{ig}}\right)$ is slightly decreased and the total smoke produced (TSP) is slightly increased, the average mass loss rate (AMLR) is drastically reduced. The peak of heat release rate (PHRR) behaves similar to the AMLR, while the total heat released (THR) is about constant. The PHRR is reduced by $47 \%$

Table 3 Cone calorimeter data

\begin{tabular}{|c|c|c|c|c|c|c|c|}
\hline & $\mathrm{PHRR}^{a} / \mathrm{kW} \mathrm{m}^{-2}$ & $\Delta$ PHRR $(\%)$ & $\mathrm{THR} / \mathrm{MJ} \mathrm{m}^{-2}$ & $\mathrm{TSP} / \mathrm{m}^{2} \mathrm{~m}^{-2}$ & $\mathrm{AMLR} / \mathrm{g} \mathrm{s}^{-1} \mathrm{~cm}^{-2}$ & $t_{\mathrm{ig}} / \mathrm{s}$ & $t_{\mathrm{PHRR}} / \mathrm{s}$ \\
\hline PS control & $1145 \pm 21$ & - & $94.8 \pm 5.9$ & $3232 \pm 256$ & $24.9 \pm 4.5$ & $40 \pm 2$ & $128 \pm 4$ \\
\hline PS nanocomposite (solution) & $665 \pm 68$ & 47 & $95.7 \pm 6.8$ & $3982 \pm 174$ & $17.8 \pm 0.5$ & $26 \pm 4$ & $48 \pm 4$ \\
\hline
\end{tabular}



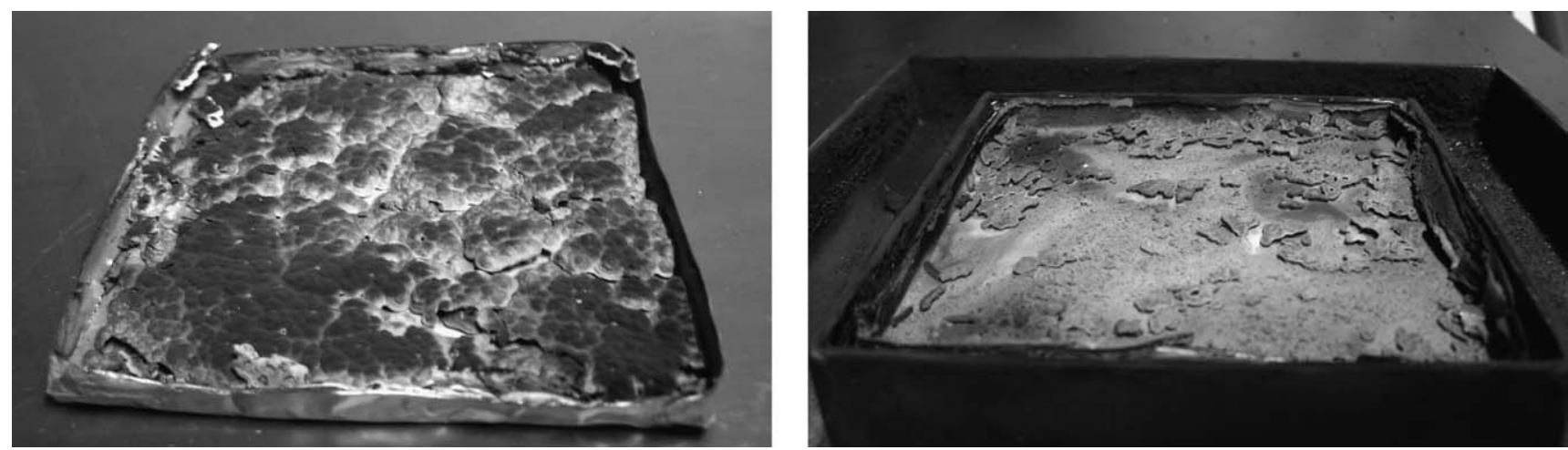

Fig. 9 Comparison of the char residues of the solution blended (left) and the melt blended (right) PS surface modified K-hectorite nanocomposite.

for the solution blended nanocomposite. This value is comparable or even slightly better than many other published PS layered silicate nanocomposites ${ }^{\mathbf{1 0}}$ with a comparable filler content. Therefore, it is presumed that the flame retardant mechanism of layered silicates is operative for the surface modified K-hectorite filler.

The difference in reduction of PHRR between the two blending methods can be explained by the homogeneity of the dispersion in the PS matrix. In contrast to the solution blended nanocomposite, the melt blended nanocomposite shows agglomerates, which cannot be destroyed completely during the melt mixing. We consider drying and low shear energy as the main problem for the complete disaggregation of the dried filler. Therefore, the specific interface between matrix and filler and hence the homogeneity of the surface layer formed during the burning of the sample are lower as compared to solution blended samples. The different quality in dispersion of the fillers with the two blending methods can also be monitored by char formation. Fig. 9 shows the residues of the two nanocomposite samples. The homogeneous char of the solution blended nanocomposite is obvious in the photograph on the left. The char surface of the solution blended sample shows almost no cracks, whereas the melt blended nanocomposite exhibits only small areas where char is formed (right image). The distribution of the filler particles is crucial for flame retardancy of layered silicate nanocomposites and drying of the clay leads to agglomeration, which changes the nanocomposite morphology.

\section{Conclusions}

A novel filler, based on a high aspect ratio, mica-like layered silicate, was synthesized from a Na-fluoro-hectorite showing a layer charge which must be tailored to the process. The obtained shear-stiff clay tactoids were exclusively modified on the external surface of the platelets by an oligomeric modifier. The resulting surface modified K-hectorite filler with aspect ratios up to 350 is white in color, contains a low amount of organic modifier $(12.5 \mathrm{wt} \%)$, and the performance of the obtained PS nanocomposites is better than that of natural clay PS nanocomposites regarding thermal and fire properties.
Consequently, this route offers a new clay based nanocomposite filler, which can be tailored for different applications.

\section{Acknowledgements}

The authors thank Dr R. Giesa, department of Macromolecular Chemistry I, University of Bayreuth, for his assistance in building the test specimen. M.R.S. and T.L. acknowledge financial support from Deutsche Forschungsgemeinschaft (SFB 840) and the Bavarian Elite Support Program.

\section{Notes and references}

1 Y. Kojima, A. Usuki, M. Kawasumi, A. Okada, T. Kurauchi and O. Kamigaito, J. Polym. Sci., Part A: Polym. Chem., 1993, 31, 983986

2 A. Usuki, Y. Kojima, M. Kawasumi, A. Okada, Y. Fukushima, T. Kurauchi and O. Kamigaito, J. Mater. Res., 1993, 8, 1179-1184.

3 R. Krishnamoorti and R. A. Vaia, Polymer Nanocomposites: Synthesis, Characterization and Modeling, American Chemical Society, Washington, DC, 2002.

4 D. Y. Wang, J. Zhu, Q. Yao and C. A. Wilkie, Chem. Mater., 2002, 14, 3837-3843.

5 N. Sheng, M. C. Boyce, D. M. Parks, G. C. Rutledge, J. I. Abes and R. E. Cohen, Polymer, 2004, 45, 487-506.

6 M. W. Möller, U. A. Handge, D. A. Kunz, T. Lunkenbein, V. Altstädt and J. Breu, ACS Nano, 2010, 4, 717-724.

7 A. B. Morgan and C. A. Wilkie, Flame Retardant Polymer Nanocomposites, Wiley-Interscience, Hoboken, NJ, 2007.

8 S. Bourbigot, M. Le Bras, S. Duquesne and M. Rochery, Macromol. Mater. Eng., 2004, 289, 499-511.

9 J. W. Gilman, C. L. Jackson, A. B. Morgan, R. Harris, E. Manias, E. P. Giannelis, M. Wuthenow, D. Hilton and S. H. Phillips, Chem. Mater., 2000, 12, 1866-1873.

10 P. Kiliaris and C. D. Papaspyrides, Prog. Polym. Sci., 2010, 35, 902958.

11 E. Manias, Nat. Mater., 2007, 6, 9-11.

12 K. Tamura, S. Yokoyama, C. S. Pascua and H. Yamada, Chem. Mater., 2008, 20, 2242-2246.

13 H. Kalo, M. W. Möller, M. Ziadeh, D. Dolejs and J. Breu, Appl. Clay Sci., 2010, 48, 39-45.

14 K. A. Carrado, A. Decarreau, S. Petit, F. Bergaya and G. Lagaly, in Handbook of Clay Science, ed. F. Bergaya, B. K. G. Theng and G. Lagaly, Elsevier Ltd., Amsterdam, 1st edn, 2006, vol. 1, ch. 4, pp. $115-139$.

15 J. Zhu, A. B. Morgan, F. J. Lamelas and C. A. Wilkie, Chem. Mater., 2001, 13, 3774-3780. 